\title{
Stroke and Mesenchymal Stromal Cells: Mechanisms of Neuroprotection and Future Prospects
}

\section{Marta Dossena *}

Cellular Neurobiology Laboratory, Department of Cerebrovascular Disease, Fondazione IRCCS Istituto Neurologico Carlo Besta, Milan, Italy

*Corresponding author: Marta Dossena, Cellular Neurobiology Laboratory, Department of Cerebrovascular Disease, Fondazione IRCCS Istituto Neurologico Carlo Besta, Milan, Italy, Tel: 0223942414; E-mail: marta.dossena@istituto-besta.it

Received date: February 24, 2016; Accepted date: February 26, 2016; Published date: March 2, 2016

Copyright: ( 2016 Dossena M. This is an open-access article distributed under the terms of the Creative Commons Attribution License, which permits unrestricted use, distribution, and reproduction in any medium, provided the original author and source are credited.

\section{Editorial}

Stroke is the second cause of morbidity and mortality worldwide [1]. The reduced blood flow generates ischemia area due to multiple processes such as excitotoxicity, ionic imbalance, depolarization of the peri-infarcted area, oxidative stress, inflammation and apoptosis, leading to neuronal death [2]. Mitochondria exert a crucial role in antioxidant cell defense; various studies related mitochondrial dysfunction to the pathophysiology of acute neurologic deficit in cerebral ischemia [3]. The lack of oxygen and glucose supply to the brain after stroke results in a drastic reduction in mitochondrial ATP production triggering necrotic cell death mechanisms. Further, reactive oxygen species (ROS) generation by mitochondria plays a critical role in neuronal damage. Excessive ROS can be scavenged by the action of antioxidant enzymes including superoxide dismutase, catalase and cytosolic and mitochondrial glutathione peroxidase [4]. However, in the case of huge ROS overproduction as observed during reperfusion, endogenous antioxidant capacity is quickly depleted [5], further inducing oxidative damage of cell macromolecules, cell damage and death [6].

To date the only pharmacological treatment currently approved for stroke is based upon systemic thrombolysis by i.v. injection of tissue plasminogen activator ( $\mathrm{t}-\mathrm{PA}$ ). Unfortunately, less than $5 \%$ of all patients can be treated with t-PA because of drug adverse effects and the narrow therapeutic window $(<4.5$ hours) [7]. Despite extensive preclinical studies, little progress has been made toward the development of new effective therapies so that the current mantra in stroke treatment is "time is brain". An urgent need is to identify and characterize a therapeutic approach directly aimed at preserving neuronal viability by specifically targeting key pathogenic pathways in brain ischemia. Recently the enormous potential of cell transplantation therapy for stroke have been highlighted. The absence of ethical concerns and the good results found in experimental animal models make mesenchymal stromal cells (MSCs) one of the most promising types of stem cells for translational applications. Although bone marrow was the first source to be identified [8], MSCs have been successfully isolated from adipose tissue, pancreas, liver, skeletal muscle, dermis, synovial membrane, and trabecular bone [9]. In vitroexpanded MSCs also have remarkable properties due to their capacity to produce a variety of growth factors, cytokines, chemokines and proteases that likely could play immunomodulatory roles after their migration into inflamed regions [10].

Recently many evidences have shown the efficacy of MSCs treatment both in in vitro [11] and in vivo models of ischemia [12]. Treatment, in vitro, with MSCs protected a neuronal cell line against oxygen-glucose deprivation damage through the secretion of vascular endothelial growth factor and interleukin-6 (IL-6). The application of
MSCs induce neuroprotection through NFkB activation leading an increase of IL-6 production that causes a decrease in apoptosis independently of the activation PI3K/Akt pathway. On the other hand IL-6 induces STAT3 and mitochondrial superoxide dismutase 2 phosphorilation, that resulted in decreased apoptosis and oxidative stress [11].

Brain-derived neurotrophic factor (BDNF) is another growth factor secreted by the MSCs that can activates a lot of pathways crucial for the survival-promoting effect in neurons, Wilkins et al demonstrate that BDNF content, in MSCs conditioned medium, diminish neuronal death by the induction of Akt phosphorilation and, at the same time, by decreasing the phosphorylation of $\mathrm{p} 38$, which is involved in the mechanisms of cell death [13]. Moreover Moriyama et al describe how the MSCs, entered into ischemic environment, are subjected to oxidative stress. This stress causes an increase in the p38 phosphorylation and, consequently, an augmented release of fibroblast growth factor 2 and bone morphogenetic protein 2 . These two growth factors activated the MEK/ERK cascade and Smad 1/5/8, respectively, bringing to an increased neurite growth [14].

It has been also demonstrated, in vivo, that subcutaneous administration of MSCs increased survival in stroke-prone spontaneously hypertensive rats, with increased levels of the antiapoptotic gene Bcl-2 and decreased superoxide production in the ischemic area [12]. MSCs neuroprotective effect could act either on apoptosis and autophagy:

Recently, MSCs retro-orbital injection reduced autophagy with reduction of Beclin-1 and LC3II expression in neurons and promoted axonal regeneration in a spinal cord ischemia/reperfusion injury model [15].

The MSCs, or their derivatives, may represent a new frontier for the treatment of stroke. To date 13 small clinical trials have been carried out, showing that stem cell therapy may be safe and technically feasible in stroke patients.

Only a deeper knowledge of the MSCs mechanism of action as antioxidants could lead to the development of innovative and effective drugs that targets this pathway in the cure of stroke.

\section{References}

1. Dulamea AO (2015) The potential use of mesenchymal stem cells in stroke therapy-From bench to bedside. J Neurol Sci 352: 1-11.

2. Mergenthaler P, Dirnagl U, Meisel A (2004) Pathophysiology of stroke: lessons from animal models. Metab Brain Dis 19: 151-167.

3. Anne Stetler R, Leak RK, Gao Y, Chen J (2013) The dynamics of the mitochondrial organelle as a potential therapeutic target. J Cereb Blood Flow Metab 33: 22-32. 
Citation: Dossena M (2016) Stroke and Mesenchymal Stromal Cells: Mechanisms of Neuroprotection and Future Prospects. J Biomol Res Ther 5: e145. doi:10.4172/2167-7956.1000e145

Page 2 of 2

4. Matés JM (2000) Effects of antioxidant enzymes in the molecular control of reactive oxygen species toxicology. Toxicology 153: 83-104.

5. Saito A, Maier CM, Narasimhan P, Nishi T, Song YS, et al. (2005) Oxidative stress and neuronal death / survival signaling in cerebral ischemia. Mol Neurobiol 31: 105-116.

6. Schild L, Reiser G (2005) Oxidative stress is involved in the Permeabilization of the inner membrane of brain mitochondria exposed to hypoxia/reoxygenation and low micromolar $\mathrm{Ca}^{2+}$. FEBS J 272: 3593-3601.

7. Cronin CA (2010) Intravenous tissue plasminogen activator for stroke: a review of the ECASS III results in relation to prior clinical trials. J Emerg Med 38: 99-105.

8. Pittenger MF, Mackay AM, Beck SC, Jaiswal RK, Douglas R, et al.(1999) Multilineage potential of adult human mesenchymal stem cells. Science 284: 143-147.

9. Baksh D, Song L, Tuan RS (2004) Adult mesenchymal stem cells: characterization, differentiation, and application in cell and gene therapy. J Cell Mol Med 8: 301-316.

10. Kassis I, Vaknin-Dembinsky A, Karussis D (2011) Bone marrow mesenchymal stem cells: agents of immunomodulation and neuroprotection. Curr Stem Cell Res Ther 6: 63-68.
11. Huang P, Gebhart N, Richelson E, Brott TG, Meschia JF, et al. (2014) Mechanism of mesenchymal stem cell-induced neuron recovery and antiinflammation. Cytotherapy 16: 1336-1344.

12. Calió ML, Marinho DS, Ko GM, Ribeiro RR, Carbonel AF, et al. (2014) Transplantation of bone marrow mesenchymal stem cells decreases oxidative stress, apoptosis, and hippocampal damage in brain of a spontaneous stroke model. Free Radic Biol Med 70: 141-154.

13. Wilkins A, Kemp K, Ginty M, Hares K, Mallam E (2009) Human bone marrow-derived mesenchymal stem cells secrete brain-derived neurotrophic factor which promotes neuronal survival in vitro. Stem Cell Res 3: 63-70.

14. Moriyama M, Moriyama H, Ueda A, Nishibata Y, Okura H, et al. (2012) Human adipose tissue-derived multilineage progenitor cells exposed to oxidative stress induce neurite outgrowth in PC12 cells through p38 MAPK signaling. BMC Cell Biol 13: 21.

15. Yin F, Meng C, Lu R, Li L, Zhang Y, et al. (2014) Bone marrow mesenchymal stem cells repair spinal cord ischemia / reperfusion injury by promoting axonal growth and anti-autophagy. Neural Regen Res 9: 1665-1671. 